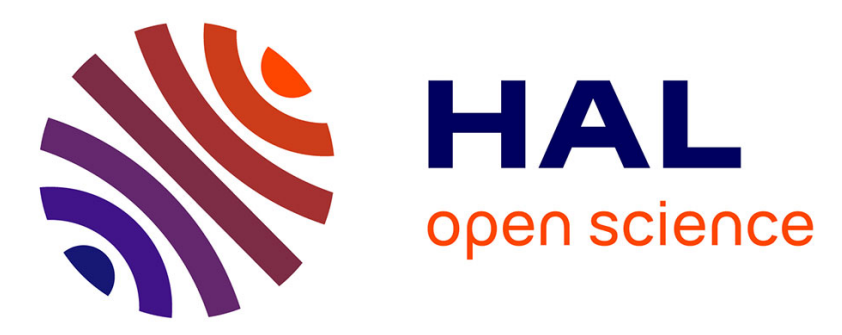

\title{
Estimation of external contact loads using an inverse dynamics and optimization approach: general method and application to sit-to-stand maneuvers.
}

Thomas Robert, Julien Causse, Gérard Monnier

\section{- To cite this version:}

Thomas Robert, Julien Causse, Gérard Monnier. Estimation of external contact loads using an inverse dynamics and optimization approach: general method and application to sit-to-stand maneuvers.. Journal of Biomechanics, 2013, 46 (13), 2220-2227 pp. 10.1016/j.jbiomech.2013.06.037 . hal-02072691

\section{HAL Id: hal-02072691 \\ https://hal.science/hal-02072691}

Submitted on 19 Mar 2019

HAL is a multi-disciplinary open access archive for the deposit and dissemination of scientific research documents, whether they are published or not. The documents may come from teaching and research institutions in France or abroad, or from public or private research centers.
L'archive ouverte pluridisciplinaire HAL, est destinée au dépôt et à la diffusion de documents scientifiques de niveau recherche, publiés ou non, émanant des établissements d'enseignement et de recherche français ou étrangers, des laboratoires publics ou privés. 


\title{
Estimation of external contact loads using an inverse dynamics and optimization approach: general method and application to sit-to-stand maneuvers.
}

\author{
T. Robert ${ }^{\mathrm{a}, *}$, J. Causse ${ }^{\mathrm{a}, \mathrm{b}}$, G. Monnier ${ }^{\mathrm{a}, \mathrm{c}}$ \\ ${ }^{a}$ Université de Lyon, F-69622, Lyon, France \\ IFSTTAR, LBMC, F-69675 Bron \\ Université Lyon 1, LBMC, F-69373 Lyon \\ ${ }^{b}$ PSA Peugeot-Citroen, France \\ ${ }^{c}$ In-Motion, France
}

\begin{abstract}
This paper presents a general method to estimate unmeasured external contact loads (ECL) acting on a system whose kinematics and inertial properties are known. This method is dedicated to under-determinate problems, e.g. when the system has two or more unmeasured external contact wrenches. It is based on inverse dynamics and a quadratic optimization, and is therefore relatively simple, computationally cost effective and robust. Net joint loads (NJL) are included as variables of the problem, and thus could be estimated in the same procedure as the ECL and be used within the cost function.

The proposed method is tested on human sit-to-stand maneuvers performed holding an handle with one hand, i.e. asymmetrical movements with multiples external contacts. Three sets of measured and unmeasured contact load components and three cost functions are considered and simulated results are compared to experimental data. For the population and movement studied, better results are obtained for a least square sharing between actuated degrees of freedom of the relative motor torques (motor torques normalized by the maximal torque production capacity). Moreover, the number of unknown ECL components does not significantly influence the results. In particular, measuring only the vertical
\end{abstract}

\footnotetext{
* Corresponding author

Email address: thomas.robert@ifsttar.fr (T. Robert)
} 
force under the seat lead to a relatively correct estimation of the ECL and NJT (RMS differences about $10 \%$ and $20 \%$ of the amplitude for the ECL and the NJT respectively), and to observe the influence of an experimental parameter (the Seat Height).

Keywords:

Inverse Dynamics, Under-determinate problem, External contact loads, Joint loads, Optimization, Sit to stand 


\section{Introduction}

Human motion analysis focuses more and more on dynamic variables. In particular, analysis of the external contact loads (ECL) allows characterizing the nature and the role of interactions between a subject and its environment. For

5 example, Chateauroux and Wang (2010) classified hand/vehicle contacts during car ingress/egress motions based on their role on the motion: exploration of the environment, balance maintenance and participation to the displacement.

Knowledge of the ECL is also necessary to analyze the dynamics of a motion, typically the internal loads such as the net joint torques (NJT) or muscle forces. These internal loads are not directly measurable with non invasive techniques. They are usually estimated using inverse dynamic approaches, i.e. based on the knowledge of the system's kinematics, its inertia properties and the ECL acting on it (e.g. Dariush et al., 2000; Doriot and Cheze, 2004; Dumas et al., 2007b; Silva and Ambrosio, 2002; Winter, 1990).

15 Classically ECL are directly recorded using load sensors. However, direct measurement may be technically complex, especially for ambulatory or "out of the lab" experiments. Besides, use of force sensors tends to reduce the "ecological" aspect of an experiment. A classical illustration would be the force plate targeting problem in gait analyses (Challis, 2001; Oggero et al., 1998). Another example would be ergonomic experiments performed in complex environments, such as car ingress-egress (Causse et al., 2009; Kim and Lee, 2009) where the difficulty to equip every mock-up parts lead experimenters to limit the possible contact areas for the subject, and eventually limit the variety and naturalness of movements.

It is therefore necessary to use modeling techniques to indirectly estimate the ECL from the known kinematics of body segments (measured or simulated). One of the method could be to use a detailed model of the contacts, in particular for the foot-ground contact during locomotion (Fluit et al., 2012; Pandy, 2001). However such models are difficult to adjust, potentially expensive in computa-

so tion and sensitive to the modeling hypotheses (Dorn et al., 2012). An alternative 
is to use an inverse approach, i.e. to estimate the ECL acting on a system that lead to its observed motion (Pillet et al., 2010; Sanders et al., 1991). However, one of the limitations of this approach is that it becomes under-determined if there are more unknown ECL components than independent governing equations. This usually occurs when there are multiple unmeasured contacts loads with the environment. Thus, setting an assumption on the load sharing becomes necessary.

In the past, only few studies have proposed to deal with these types of underdetermined problems. Most of them focused on bipedal phase of the gait and

40 represented the load sharing with mathematical functions (Davis and Cavanagh, 1993; Hardt and Mann, 1980; Koopman et al., 1995; Ren et al., 2008). While these methods provided satisfying results, they were very specific to the given task and the population. A more generic approach for solving the load sharing problem consists by minimizing a cost function was presented by (Vaughan 45 et al., 1982). Instead of focusing only on the ECL problem, Vaughan et al. (1982) chose to explicitly introduce the unknown net joint loads (NJL) into the problem. It allowed to estimate NJL and ECL in the same procedure and also to use NJL into the cost function. Based on this approach, they obtained interesting results for different kind of movements. However, the study suffered

so from several limitation: there results were strongly dependent on the initial guess of the solution, the methods was only tested in $2 \mathrm{D}$ and in cases with no more than two unknown ECL wrenches, and the results were reported for only one cost function.

This indicates that there is still a lack of methodologies for estimating the

55 ECL, particularly in under-determined cases. This study proposes a generic method which utilizes a combination of inverse dynamics and optimization. It is intended to be relatively simple, computationally cost effective and robust. The proposed method is tested on human sit-to-stand maneuvers performed holding an handle with one hand, i.e. asymmetrical movements with multiples 6o external contacts. Influence of the number of unknown ECL components and of different cost functions are evaluated. 


\section{Modeling}

This section introduces the generic problem to be solved in this study.

\subsection{Description of system considered}

The system considered is a whole body human model made of $n_{s}$ rigid segments linked by $n_{j}$ joints representing a total of $n_{\text {dof }}$ degrees of freedom (DoF). The kinematics and inertia properties of the segments are considered to be known.

\subsection{Dynamics and constraints of the system}

The dynamic wrenches for each segments, due to the inertial and gravitational effects, can be estimated from their kinematics and inertia properties and aggregated to obtain the dynamic wrench of the overall system. This wrench has to be balanced by the known and unknown ECL, resulting in a system of 6 linear equations with $n_{u}$ unknowns. Alternatively, we chose to write the

75 Newton-Euler equations for each segments, introducing the internal loads acting between the segments. It led to a set of $6 n_{s}$ linear equations ( 6 per segments) with $n_{u}+6 n_{j}$ unknowns (corresponding to the unknown ECL components and the NJL):

$$
a_{e q} x=b_{e q}
$$

were $x=\left[\begin{array}{ll}S U\end{array}\right]^{t}$ is a single vector grouping all the unknowns, $S$ is the vectors containing the $6 n_{j}$ NJL ( 3 forces and 3 torques per joint), $U$ is the vectors of the $n_{u}$ unknown ECL components, $a_{e q}$ is a matrix relating the joints and unknown ECL components with the corresponding affected segments, and $b_{e q}$ is the effect of the dynamic wrench, known ECL and loads due to the gravity for all the segments (see Appendix for the details).

85 The physical limitations of the studied system, such as unilaterality of the contacts or maximum motor torques that could be developed, are also considered in this study. In order to make the resolution of the problem faster and more robust( see Section 2.3), we chose to express these limitations as linear equations 
of the unknown vector $x$. Mathematical description of these constraints are 9o given in Appendix.

The description of the system dynamics and limitations boils down to the linear system:

$$
\left\{\begin{array}{l}
a_{e q} x=b_{e q} \\
a_{c} x \leq b_{c}
\end{array}\right.
$$

\subsection{Resolution}

This study focuses on under-determined problems for which there are fewer governing equations than the unknowns $\left(6 n_{s}<6 n_{j}+n_{u}\right)$. In order to select only one solution among all the possibles, additional expectations for this solution are introduced via a function to be minimized. For numerical purposes (developed in the discussion) we propose: 1) to write this function in a specific form (squared euclidean norm of a linear function of $x$ ), 2) to add an additional term to it - the squared euclidean norm of $x$ with a very low weighting factor - and 3) to set the equality constraints as penalties. Eventually, it led to the following optimization problem:

$$
\min _{x} \alpha\|s x\|^{2}+\beta\|x\|^{2}+\gamma\left\|a_{e q} x-b_{e q}\right\|^{2}, \text { s.t. } \quad a_{c} x \leq b_{c}
$$

where \|\| represents the euclidean norm, $s$ is a matrix defining a linear transformation of $x$, and $\alpha, \beta$ and $\gamma$ are weighting factors.

This problem can be rewritten as a quadratic programming problem:

$$
\min _{x} \frac{1}{2} x^{T} H x+g^{T} x, \text { s.t. } \quad a_{c} x \leq b_{c}
$$

with $H=2 \alpha s^{T} s+\beta I+\gamma a_{e q}^{T} a_{e q}$ a positive definite matrix (see Discussion) and $g^{T}=-2 \gamma b_{e q}^{T} a_{e q}$. 


\section{Case Study}

\subsection{Task and motions studied}

110

Experimental data were collected on six healthy young male volunteers (stature $=1752 \pm 61 \mathrm{~mm}$, weight $=66 \pm 8 \mathrm{~kg}$ and age $=26 \pm 3$ yo), having no musculoskeletal disorder.

Subjects were initially seated on a flat rigid seat with feet on the ground and the right hand grasping a horizontal handle. The handle was centered in the sagittal plane of the subject, located at the level of the subject's eyes when seated and at a distance corresponding to the shoulder-wrist length. They were asked to rise from the seat to a standing posture using the handle (see Figure 1). Three different seat heights were tested: $50 \%\left(H_{50}\right), 75 \%\left(H_{75}\right)$ or $100 \%$ $\left(H_{100}\right)$ of the knee height. The task was repeated two times for $H_{50}$ and $H_{100}$ 120 and six times for $H_{75}$.

\subsection{Experimental Data Collection and Processing}

A Motion Analysis ${ }^{\circledR}$ system, sampling at $100 \mathrm{~Hz}$, was used to capture the trajectories of 51 reflective markers placed on the subject. All ECL between the subject and its environment were recorded using three force plates (under each foot and under the seat) and a 6-axes load sensor placed between the handle and the frame.

Kinematics was reconstructed using a global optimization procedure (Wang et al., 2005) and a 16 rigid bodies - 33 degrees of freedom kinematic model (see Figure 2). The segment inertia parameters were estimated from regression equations (Dumas et al., 2007a). NJL were computed using a recursive approach (Doriot and Cheze, 2004) and two calculation strategies (bottom-up and top-down) converging at the lumbar joint (Robert et al., 2007). These NJL computed using all the contact loads information will later be used as reference to estimate the quality of the optimization (see Section 3.5). 
The methodology proposed in section 2 is applied using the measured kinematics and different subsets of the measured ECL.

Three different sets of known (i.e. which are measured) and unknown ECL were considered (see Figure 2): 1) $S_{1}$ - both seat and hand contact wrenches are known; 2) $S_{2}$ - only seat contact wrench is known; 3) $S_{3}$ - only the vertical force of seat contact wrench is known and other components are assumed absent.

When unknown, hand to handle interaction was represented by a full wrench applied at the projection of the third metacarpophalangeal joint on the handle. The contact between the foot and the ground were modeled as 3 punctual unilat145 eral contacts with tangential force limited by friction (static coefficient $\mu=0.5$ ) and positioned under the 1st and the 5th metatarsal heads and the heel. These 3 punctual contact wrenches were further aggregated to represent the foot to ground interaction with a single contact wrench.

Figure 2 about here.

Three types of constraints were applied: unilaterality and dry friction constraints for punctual contacts, and maximal motor torques that could be developed by the subject (see Table 1). Mathematical description of these constraints are given in Appendix.

Table 1 about here.

\subsection{Resolution of the Optimization Problem}

Three different expectations on the load sharing were tested: minimization in a least square sense of 1 ) the unknown external contact forces (ECF); 2) the net joint torques (NJT), 3) the motor torques normalized by their maximum allowable value ( $\mathrm{MT}_{\%}$ see Table 1$)$. It resulted in three cost functions $C_{i}$ 's of the form:

$$
C_{i}(x)=\alpha_{i}\left\|s_{i} x\right\|^{2}+\beta\|x\|^{2}+\gamma\left\|a_{e q} x-b_{e q}\right\|^{2}
$$


with $s_{i}$ matrices defining the linear transformation of $x$ - the unknown vector of ECL and NJL - into the vector of ECF, NJT or MT\% respectively (details in Appendix). Values of $\alpha_{i}$ were adjusted based on the average value of $s_{i}$ (see Table 2), while $\beta$ and $\gamma$ were set to $10^{-5}$ and $10^{5}$ respectively.

These quadratic programming problems were solved using the Matlab ${ }^{\circledR}$ quadprog solver. The algorithm was automatically initialized for the first frame of the motion (initial point set to a vectors of ones (MathWorks, 2012)). For the remaining frames it was initialized using the results of the previous frame.

\subsection{Analyses}

Three cost functions $\left(C_{1}-C_{3}\right)$, three contact configurations $\left(S_{1}-S_{3}\right)$ and three experimental seat heights $\left(H_{50}, H_{75}\right.$ and $\left.H_{100}\right)$ were considered. Seven combinations of these variables were tested (see Table 2). For each of them, the NJL and the unknown ECL were estimated for every maneuver performed by the six subjects. Forces and torques were normalized by body weight (BW) 175 and body weight times body height (BW.BH) respectively (Hof, 1996). Peak values of the norm of the simulated ECL and NJT were extracted and referred as $P_{\text {sim }}$ hereafter. In addition, time history profiles of the norm of the simulated data were compared to their reference curves. RMS differences normalized by the amplitude of the reference curve were computed and referred as $R_{\%}$. The reference data were: 1) the measured experimental data for the ECL; 2) the results of the inverse dynamics performed using the experimentally measured ECL (see section 3.2) for the NJT.

ANOVAs and Tukey's post-hoc tests were used to analyze the effects of 1) the cost function on $R_{\%}$ in $S_{2}$ and $H_{75}$ (three first rows of Table 2), 2) the contact configuration on $R_{\%}$ using $C_{3}$ and in $H_{75}$ (rows three to six of Table 2), and 3) the seat height on $P_{\text {sim }}$ using $C_{3}$ and in $S_{3}$ (last three rows of Table 2).

\section{Results}

The procedure successfully converged for all the tested motions and configurations. An evaluation was performed to observe if equality constraints set as 
penalties were satisfied. It was found that the residual values of $\left\|a_{e q} \cdot x-b_{e q}\right\|^{2}$ (see Equation (5))remained negligible (less than $10^{-4}$ ) in all cases, except for some motions in contact the configuration $S_{1}$, where it reached up to 3 N.m around the seat-off for torque constraints along the transverse axis.

Figure 3 displays an example of reference data (measured ECL and NJT obtained through inverse dynamics with the measured ECL) for a representative subject in configuration $S_{2}$ and $H_{75}$. These results are comparable in term of profiles and amplitude to the classical data available in the literature for sitto-stand maneuver (Bahrami et al., 2000; O'Meara and Smith, 2006). In this Figure 3, simulated results obtained in the same situation for different cost functions are also superimposed to the reference data. Both the shape of the simulated curves and their amplitudes are overall in good agreement with the reference ones.

An example of simulated and measured center of pressure trajectories under each foot is shown in Figure 4. The loaded area are comparable. In this particular case the experimental trajectory goes outside the modeled base of support (BoS) for some frames of the motion, whereas the simulated trajectory remains inside.

Figure 5 displays the normalized RMS differences between the simulated and reference data $\left(R_{\%}\right)$ averaged accross trials and subjects for each contact and joints and the three cost functions tested (contact configuration $S_{2}$ and seat height $H_{75}$ ). Very high values for the right wrist NJT are due to very small amplitudes of the reference curves. Therefore, they are discarded in further analyses. Overall, $R_{\%}$ are smaller for $C_{3}$ and higher for $C_{1}$. This is confirmed by an ANOVA with factors Contact and Cost function performed on the $R_{\%}$ values ${ }_{215}$ of the ECF. It showed a significant effect $(\mathrm{p}<0.05)$ of the Cost function, the Contact and their interactions. Post-hoc tests showed that $R_{\%}$ are significantly different for each cost function, with the smallest values for $C_{3}$ and the highest for $C_{1}$. Similar results are obtained for the NJT, although $R_{\%}$ for $C_{2}$ and $C_{3}$ are not significantly different.

A similar analysis was performed to evaluate the effect of the contact config- 
uration using the cost function $C_{3}$ in seat height $H_{75}$. An ANOVA with factors Contact and Contact configuration performed on the $R_{\%}$ for the ECF showed that neither the Contact configuration nor the interaction has a significant effect ( $p=.157$ and $p=.321$, respectively). Similar results are obtained for the NJT.

Two ANOVAs with factors Contact and Seat height performed on the $P_{\text {sim }}$ for the ECF and for the NJT confirmed that the Seat height has a significant effect on the $P_{\text {sim }}$. Post-Hoc tests showed that the higher the seat the smaller the $P \operatorname{sim}\left(H_{100}<H_{75}<H_{50}\right)$.

\section{Discussion}

\subsection{Numerical Aspects}

This paper presents a general method to estimate unmeasured external contact loads acting on a system whose kinematics and inertial properties are known. This method was intended to be relatively simple and robust. For the case study considered the optimization problem converged systematically, and one could remark the smoothness of the time profile for the simulated variables, although the problem is solved frame by frame. It is notably imputable to the numerical properties of the optimization problem. We chose to write the problem as a quadratic program (quadratic cost function and linear constraints). For this type of problem, if the $\mathrm{H}$ matrix of the cost function (see equation 5) is positive definite (always non negative, nil only for $x=0$ ) and if the problem has a solution, then it exists a unique minimum that is the global minimum. The solver will thus systematically and quickly converge toward the desired solution.

The cost functions used in this study are the sum of three terms (see Equation (5)). The first and last terms are strictly non negative functions that may be equal to zero for non zero value of $x$. Adding the squared norm of $x$, even with a very small weight, ensures that the cost function can only be nil only when $x=0$, i.e. the cost functions are positive definite. In other words, the first and last terms of the cost function did not necessarily nullify the space of 
solution. Typically the foot contact model used in this study (3 contact points) induced a redundancy in the horizontal plane: an infinity of individual contact forces combinations summed up to the same overall contact force for the foot, and thus the same motor torques and the same value of the optimization criterion. The role of the second part of the criterion, slightly weighted, is then 255 to nullify the space of solution and as such to improve the stability and convergence efficiency of the optimization algorithm. Because of its small relative weight, it did not significantly changed the results in terms of the resultant of ECL or NJL.

This study focused on under-determined problems. However, in some situ260 ations, the system of equation (2) may become over-constrained. It typically happens in case of temporary inconsistencies between the modeling hypotheses and the imposed experimental data (estimated accelerations or BSIP). In this study we chose to set the equality constraints as penalties in the cost function. It led to the optimization problem (3), only constrained by the set of inequalities representing the system's limitations. These later are independent of the experimental data and, besides modeling incoherences, unlikely to nullify the space of solution. Consequently the problem (3) always has at least a solution. Although this solution only satisfies as well as possible the equations of motion, it may be acceptable for small residual values of the penalties. In this study over-constrained situations may happen around the seat-off in the contact configuration $S_{1}$. As displayed in Figure 4, the foot contact wrenches may be too tightly constrained for some motion, leading to small discrepancies between the modeled $\mathrm{BoS}$ and the experimental $\mathrm{CoP}$ trajectory. These discrepancies could be compensated by the hand contact wrench when this later is not imposed, but led to an over-constrained problem when the hand contact wrench is imposed (contact configuration $S_{1}$ ). The observed residual values remained relatively small, and the obtained solutions were thus acceptable. However, a track for improvement would be to finely defined the shape of the BoS, by adding contact points for example. 


\subsection{Case study: cost function and applicability}

The proposed methodology was applied to sit-to-stand motions with an handle. Different load sharing hypotheses were tested. Cost function $C_{3}$ provide the better results (the smaller $R_{\%}$ ). It principally implies a least square sharing between actuated DoF of the relative motor torques (motor torques normalized by the maximal torque production capacity). It therefore prevents to overload those DoF which are having small capacity. This effect is best seen on the right upper limb, where the use of $C_{3}$ tend to limit the loads along the right upper limb DoF, with smaller force production capacities relative to those of the lower limbs. This criterion is quite similar to the minimization of the individual muscle stress (indivudual muscle forces normalized by their physiological cross sectional area) classically used for solving the under-determinated muscle force sharing problem (e.g. Erdemir et al., 2007, for a review). Other criteria could have been considered, in particular considering the p-norm of $s_{i} x$ to the power $p$ with $p \neq 2$ and the min/max criterion (Rasmussen et al., 2001). It could also be interesting to extend this problem to include individual muscle forces in the criterion. It would not change the nature of the problem as, by neglecting the contraction dynamics, the muscle forces can be expressed as a linear function transformation of the motor torques via a level arm matrix.

Three different contact configurations were considered in this study. Statistical analysis showed that this parameter did not significantly influence the quality of the results (difference between reference and simulated data). In particular, reducing the number of unknowns did not improve the $R_{\%}\left(S_{1}\right.$ vs $\left.S_{2}\right)$. Results obtained in $S_{3}$ (using only the vertical force component of the seat contact wrench) are important in terms of application. They suggest that, for the studied movement and population, measuring only the vertical force under the seat would allow a relatively correct estimation of all ECL and NJT: not only the values of $R_{\%}$ were small (about $10 \%$ for the feet ECL and $20 \%$ for the NJT), but the influence of an experimental parameters (the Seat Height) was also correctly predicted. In this case, peak values of NJT and ECL increased when the seat height decreased, as observed from the experimental data (Robert et al., 2011). 
This could be of particular importance for ergonomic studies where usually the goal is to understand the influence of different experimental parameters on the motion dynamics and which often suffer from instrumentation difficulties.

\subsection{Limitations}

The methodology was only evaluated for one type of motion and a small sample of single population. Load sharing criterion may evolve between motion and population, and may include other parameters such as the comfort or balance. This should be further studied.

Moreover, the contact configurations considered in this study remained constant during the motion. Although the kinematics and measured contact loads may sufficiently drive the problem, discontinuities in solution may appear when considering intermittent contacts. One could thus consider describing the problem using parametric curves and to solve it as a whole instead of frame by frame.

\section{Conflict of interest statement}

None of the authors has any conflict of interest to report in this research.

Bahrami, F., Riener, R., Jabedar-Maralani, P., Schmidt, G., 2000. Biomechanical analysis of sit-to-stand transfer in healthy and paraplegic subjects. Clinical Biomechanics 15, 123 - 133.

Causse, J., Chateauroux, E., Monnier, G., Wang, X., Denninger, L., 2009. Dynamic analysis of car ingress/egress movement: an experimental protocol and preliminary results. SAE International Journal of Passenger Cars-Mechanical Systems 2, 1633-1640.

Chaffin, D., Andersson, G., Martin, B., 2006. Occupational Biomechanics. J. Wiley \& Sons, New York, NY. 4th edition.

Challis, J.H., 2001. The variability in running gait caused by force plate targeting. Journal of Applied Biomechanics 17, 77-83. 
Chateauroux, E., Wang, X., 2010. Car egress analysis of younger and older drivers for motion simulation. Applied Ergonomics 42, 169 - 177.

Dariush, B., Hemami, H., Parnianpour, M., 2000. A well-posed, embedded constraint representation of joint moments from kinesiological measurements. Journal of biomechanical engineering 122, 437-445.

Davis, B.L., Cavanagh, P.R., 1993. Decomposition of superimposed ground reaction forces into left and right force profiles. Journal of Biomechanics 26, $593-597$.

Delp, S.L., 1990. Surgery simulation: a computer graphics system to analyze and design musculoskeletal reconstructions of the lower limb. Ph.D. thesis. Stanford University.

Doriot, N., Cheze, L., 2004. A three-dimensional kinematic and dynamic study of the lower limb during the stance phase of gait using an homogeneous matrix approach. IEEE Trans Biomed Eng 51, 21-27.

Dorn, T.W., Lin, Y.C., Pandy, M.G., 2012. Estimates of muscle function in human gait depend on how foot-ground contact is modelled. Computer Methods in Biomechanics and Biomedical Engineering 15, 657-668.

Dumas, R., Cheze, L., Verriest, J.P., 2007a. Adjustments to mcconville et al. and young et al. body segment inertial parameters. Journal of Biomechanics $40,543-553$.

Dumas, R., Nicol, E., Cheze, L., 2007b. Influence of the 3D inverse dynamic method on the joint forces and moments during gait. Journal of Biomechanical Engineering 129, 786-790.

Erdemir, A., McLean, S., Herzog, W., van den Bogert, A.J., 2007. Model-based estimation of muscle forces exerted during movements. Clin Biomech (Bristol, Avon) 22, 131-154. 
Fluit, R., van der Krogt, M., van der Kooij, H., Verdonschot, N., Koopman, H., 2012. A simple controller for the prediction of three-dimensional gait. Journal of Biomechanics 45, 2610 - 2617.

Garner, B., Pandy, M., 2000. Musculoskeletal model of the upper limb based on the visible human male dataset. Computer methods in Biomechanics and Biomedical Engineering 4, 93-126.

Hardt, D.E., Mann, R.W., 1980. A five body - three dimensional dynamic analysis of walking. Journal of Biomechanics 13, $455-457$.

Hof, A.L., 1996. Scaling gait data to body size. Gait \& Posture 4, $222-223$.

Kim, S., Lee, K., 2009. Development of discomfort evaluation method for car ingress motion. International Journal of Automotive Technology 10, 619-627. $10.1007 / \mathrm{s} 12239-009-0073-6$.

Koopman, B., Grootenboer, H.J., de Jongh, H.J., 1995. An inverse dynamics model for the analysis, reconstruction and prediction of bipedal walking. Journal of Biomechanics 28, 1369 - 1376.

MathWorks, 2012. Optimization toolbox user's guide, matlab r2012b.

Oggero, E., Pagnacco, G., Morr, D., Simon, S., Berme, N., 1998. Probability of valid gait data acquisition using currently available force plates. Biomedical sciences instrumentation 34, 392-397.

O'Meara, D.M., Smith, R.M., 2006. The effects of unilateral grab rail assistance on the sit-to-stand performance of older aged adults. Human Movement Science $25,257-274$.

Pandy, M., 2001. Computer modeling and simulation of human movement. Annual review of biomedical engineering 3, 245-273.

Pillet, H., Bonnet, X., Lavaste, F., Skalli, W., 2010. Evaluation of force plate-less estimation of the trajectory of the centre of pressure during gait. comparison of two anthropometric models. Gait \& Posture 31, 147-152. 
Rasmussen, J., Damsgaard, M., Voigt, M., 2001. Muscle recruitment by the $\min / \max$ criterion - a comparative numerical study. J Biomech 34, 409-415.

Ren, L., Jones, R.K., Howard, D., 2008. Whole body inverse dynamics over a complete gait cycle based only on measured kinematics. J Biomech 41, $2750-2759$.

Robert, T., Causse, J., Wang, X., 2011. Dynamics of sit-to-stand motions: effect of seat height, handle use and asymmetrical motions. Computer Methods in Biomechanics and Biomedical Engineering 14, 191-192.

Robert, T., Chèze, L., Dumas, R., Verriest, J.P., 2007. Validation of net joint loads calculated by inverse dynamics in case of complex movements: application to balance recovery movements. Journal of Biomechanics 40, 2450-2456.

Sanders, R.H., Wilson, B.D., Jensen., R.K., 1991. Accuracy of derived ground reaction force curves for a rigid link human body model. Journal of Applied Biomechanics 7, 330-343.

Silva, M., Ambrosio, J., 2002. Kinematic data consistency in the inverse dynamic analysis of biomechanical systems. Multibody System Dynamics 8, 219-239.

Vaughan, C.L., Hay, J.G., Andrews, J.G., 1982. Closed loop problems in biomechanics. part ii-an optimization approach. J Biomech 15, 201-210.

Wang, X., Chevalot, N., Monnier, G., Ausejo, S., Ángel Suescun, Celigüeta, J., 2005. Validation of a model-based motion reconstruction method developed in the realman project. SAE Transactions Journal of Passenger Cars - Electronic and Electrical Systems 114, 873-879.

Winter, D., 1990. Biomechanics and Motor Control of Human Movement. Wiley, New York. 
Table 1

Table 1: Maximum torques for the different degrees of freedom of the considered model

\begin{tabular}{|c|c|c|c|}
\hline Joint & Motion & $\begin{array}{l}\text { Maximum torque } \\
\text { value (N.m) }\end{array}$ & Source \\
\hline \multirow[t]{6}{*}{ Hip Joint } & Flexion & 185 & Chaffin et al. (2006) \\
\hline & Extension & 190 & Chaffin et al. (2006) \\
\hline & Abduction & 190 & Delp (1990) \\
\hline & Adduction & 190 & Delp (1990) \\
\hline & Internal Rotation & 60 & Delp (1990) \\
\hline & External Rotation & 60 & Delp (1990) \\
\hline \multirow[t]{4}{*}{ Knee Joint } & Flexion & 100 & Chaffin et al. (2006) \\
\hline & Extension & 168 & Chaffin et al. (2006) \\
\hline & Internal Rotation & 20 & Expertize \\
\hline & External Rotation & 20 & Expertize \\
\hline \multirow[t]{4}{*}{ Ankle Joint } & Dorsiflexion & 126 & Chaffin et al. (2006) \\
\hline & Plantarflexion & 126 & Chaffin et al. (2006) \\
\hline & Inversion & 20 & Expertize \\
\hline & Eversion & 20 & Expertize \\
\hline \multirow[t]{6}{*}{ Shoulder Joint } & Flexion & 92 & Chaffin et al. (2006) \\
\hline & Extension & 67 & Chaffin et al. (2006) \\
\hline & Abduction & 71 & Chaffin et al. (2006) \\
\hline & Adduction & 67 & Chaffin et al. (2006) \\
\hline & Internal Rotation & 52 & Chaffin et al. (2006) \\
\hline & External Rotation & 33 & Chaffin et al. (2006) \\
\hline \multirow[t]{4}{*}{ Elbow Joint } & Flexion & 77 & Chaffin et al. (2006) \\
\hline & Extension & 46 & Chaffin et al. (2006) \\
\hline & Pronation & 15 & Garner and Pandy (2000) \\
\hline & Supination & 15 & Garner and Pandy (2000) \\
\hline \multirow[t]{4}{*}{ Wrist Joint } & Flexion & 185 & Garner and Pandy (2000) \\
\hline & Extension & 190 & Garner and Pandy (2000) \\
\hline & Abduction & 190 & Garner and Pandy (2000) \\
\hline & Adduction & 190 & Garner and Pandy (2000) \\
\hline \multirow[t]{3}{*}{ Lumbar Joint } & Flexion & 143 & Chaffin et al. (2006) \\
\hline & Extension & 234 & Chaffin et al. (2006) \\
\hline & Lateral Flexion & 159 & Chaffin et al. (2006) \\
\hline \multirow{2}{*}{ Neck Joint } & Flexion & 100 & Chaffin et al. (2006) \\
\hline & Extension & 100 & Chaffin et al. (2006) \\
\hline
\end{tabular}


Table 2

Table 2: The different combination of parameters tested, and the corresponding weights of the cost function.

\begin{tabular}{|c|c|c|c|c|c|c|c|}
\hline$\#$ & $\begin{array}{l}\text { Cost } \\
\text { Function }\end{array}$ & $\begin{array}{l}\text { Contact } \\
\text { Configuration }\end{array}$ & $\begin{array}{l}\text { Seat } \\
\text { Height }\end{array}$ & $\alpha_{i}$ & $\beta$ & $\gamma$ & Tested Effect \\
\hline 1 & $C_{1}$ & $S_{2}$ & $H_{75}$ & $10^{-1}$ & $10^{-5}$ & $10^{5}$ & \multirow{3}{*}{$\begin{array}{l}\text { Cost } \\
\text { Function }\end{array}$} \\
\hline 2 & $C_{2}$ & $S_{2}$ & $H_{75}$ & 1 & $10^{-5}$ & $10^{5}$ & \\
\hline 3 & $C_{3}$ & $S_{2}$ & $H_{75}$ & 500 & $10^{-5}$ & $10^{5}$ & \\
\hline 4 & $C_{3}$ & $S_{1}$ & $H_{75}$ & 500 & $10^{-5}$ & $10^{5}$ & \multirow{3}{*}{$\begin{array}{l}\text { Contact } \\
\text { Configuration }\end{array}$} \\
\hline 3 & $C_{3}$ & $S_{2}$ & $H_{75}$ & 500 & $10^{-5}$ & $10^{5}$ & \\
\hline 5 & $C_{3}$ & $S_{3}$ & $H_{75}$ & 500 & $10^{-5}$ & $10^{5}$ & \\
\hline 6 & $C_{3}$ & $S_{3}$ & $H_{50}$ & 500 & $10^{-5}$ & $10^{5}$ & \multirow{3}{*}{ Seat Height } \\
\hline 5 & $C_{3}$ & $S_{3}$ & $H_{75}$ & 500 & $10^{-5}$ & $10^{5}$ & \\
\hline 7 & $C_{3}$ & $S_{3}$ & $H_{100}$ & 500 & $10^{-5}$ & $10^{5}$ & \\
\hline
\end{tabular}


Figure 1

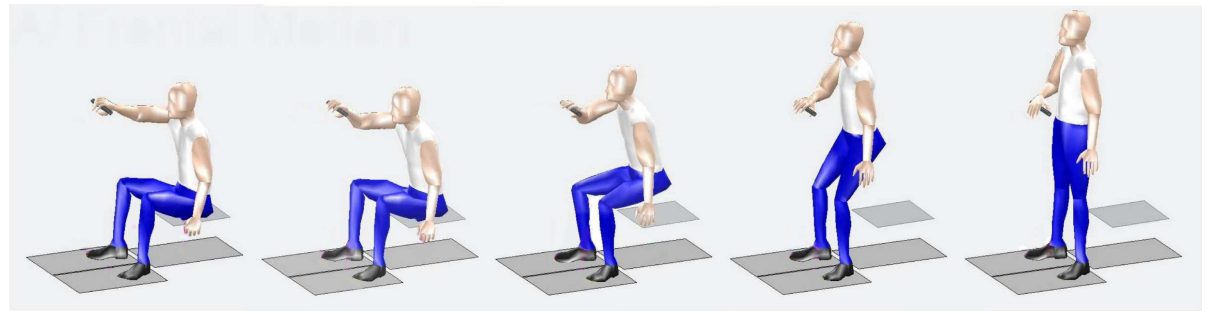

Figure 1: The sit to stand maneuver considered: example of a reconstructed motion with the seat height at $75 \%$ of the knee height. 
Figure 2

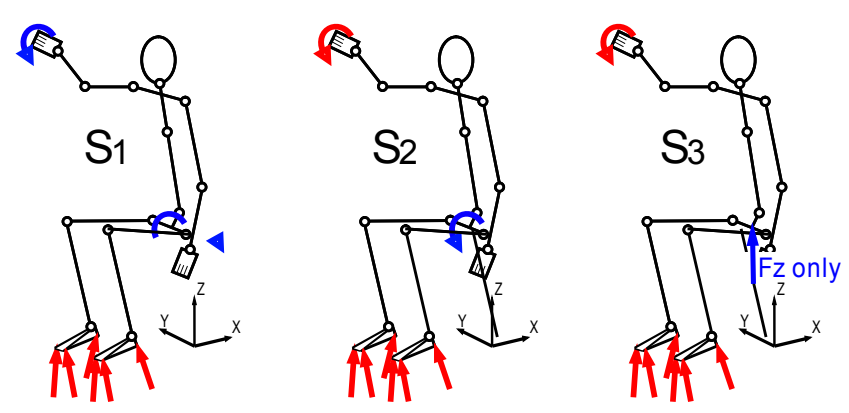

Figure 2: The multibody model considered in the three contact configurations tested. Straight stand for known and unknown contact loads respectively. 
Figure 3
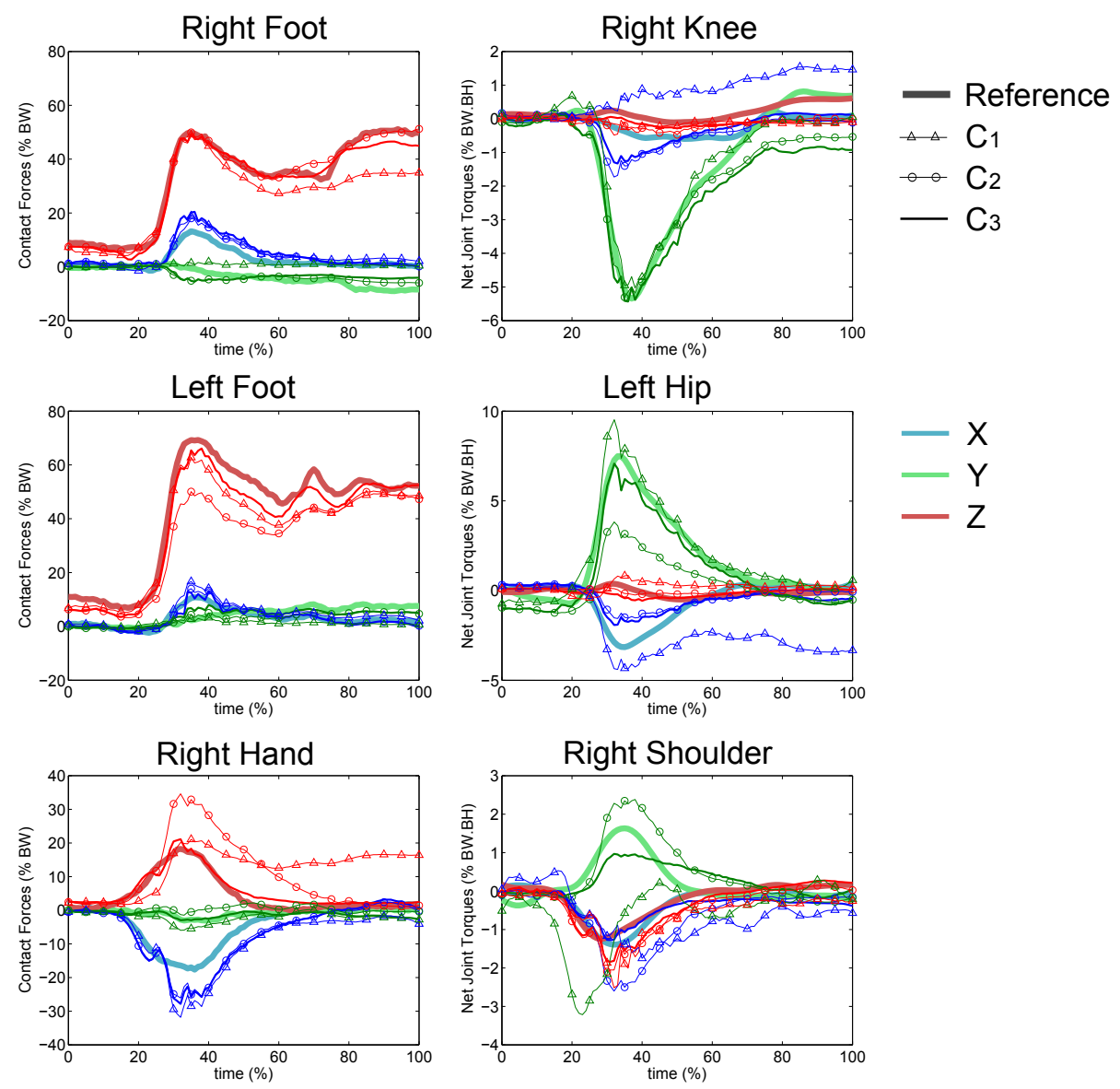

Figure 3: Example of reference and simulated contact forces and joint torques obtained for a sit-to-stand motion (seat height at $75 \%$ of the knee height, contact configuration $S 2$ ) for three different cost functions. Data are expressed in coordinate systems parallel to the Global Coordinate System. Differences among the cost functions are best seen for hand forces and shoulder torques(see last row in the figure). 
Figure 4

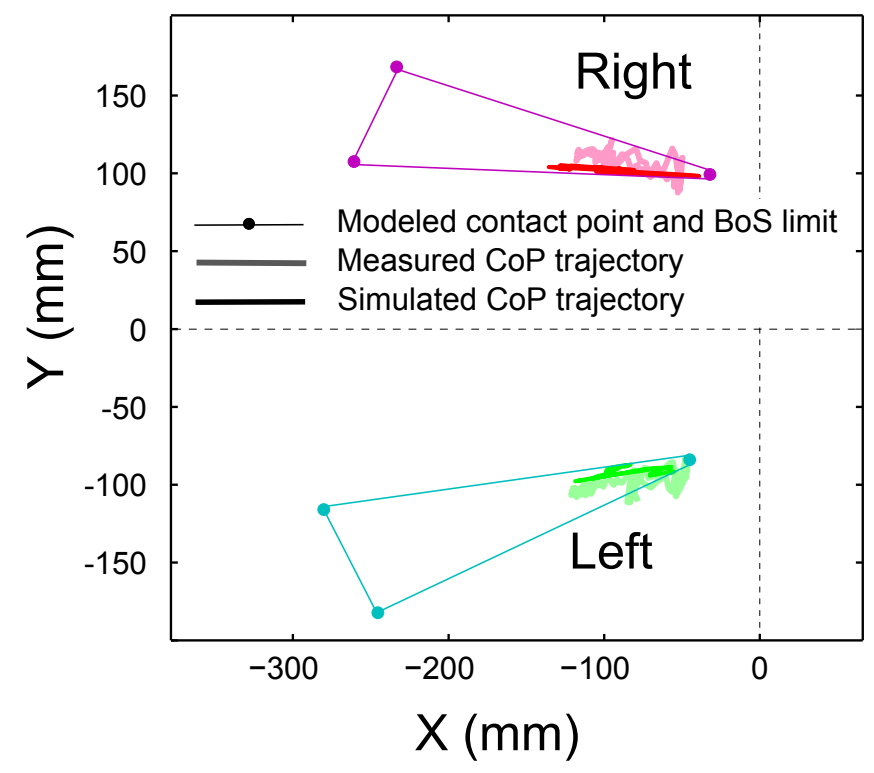

Figure 4: An example of the measured and simulated trajectories of the Center of Pressure under each foot, expressed in the global coordinate system. Note that in this case the experimental trajectories goes out of the modeled base of support, while the simulated trajectories remain inside. 
Figure 5

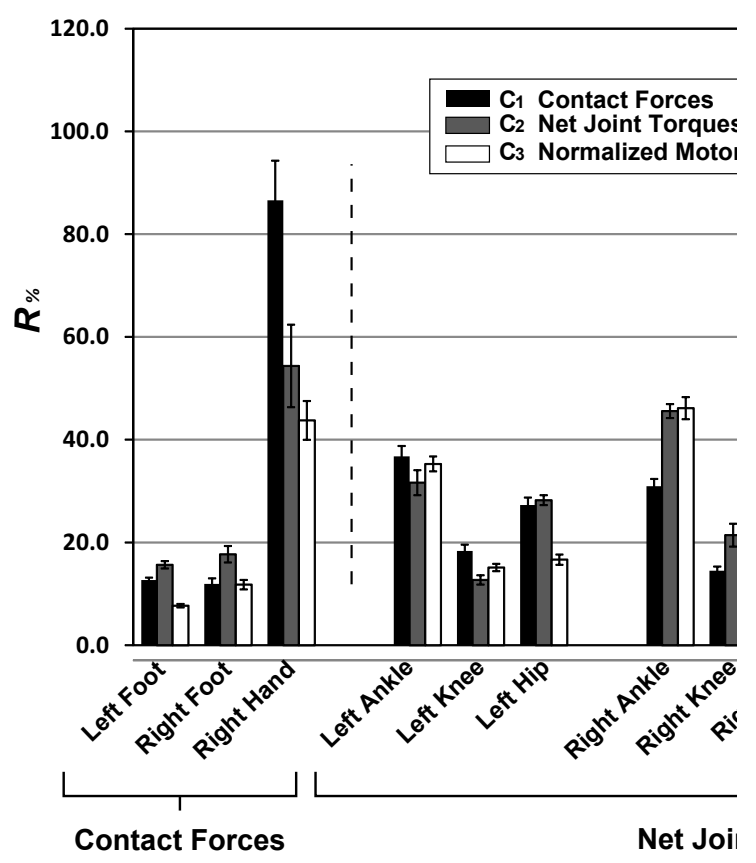

Figure 5: $R_{\%}$ (normalized RMS differences between simulated and reference data - see section 3.5 ) for different cost functions and different joint and contacts, averaged accross subjects and trials, in contact configuration $S_{2}$ and seat height $H_{75}$. Vertical bars represents \pm one standard error of the mean (SEM). Results above $120 \%$ are given in the figure. The high values for the right wrist net joint torques are due to small amplitudes of the experimental curves. 